\title{
Téoros
}

Revue de recherche en tourisme

\section{Les ghettos du gotha. Comment la bourgeoisie défend ses espaces, Michel PINÇON et Monique PINÇON-CHARLOT, Paris : Seuil, 2007, 203 pages, ASIN : B005QDPONO}

\section{Martin Drouin}

Volume 30, numéro 2, 2011

URI : https://id.erudit.org/iderudit/1012250ar

DOI : https://doi.org/10.7202/1012250ar

Aller au sommaire du numéro

Éditeur(s)

Université du Québec à Montréal

ISSN

0712-8657 (imprimé)

1923-2705 (numérique)

Découvrir la revue

Citer ce compte rendu

Drouin, M. (2011). Compte rendu de [Les ghettos du gotha. Comment la bourgeoisie défend ses espaces, Michel PINÇON et Monique PINÇON-CHARLOT, Paris : Seuil, 2007, 203 pages, ASIN : B005QDPONO]. Téoros, 30(2), 128-128.

https://doi.org/10.7202/1012250ar d'utilisation que vous pouvez consulter en ligne. 


\section{POUR EN LIRE PLUS}
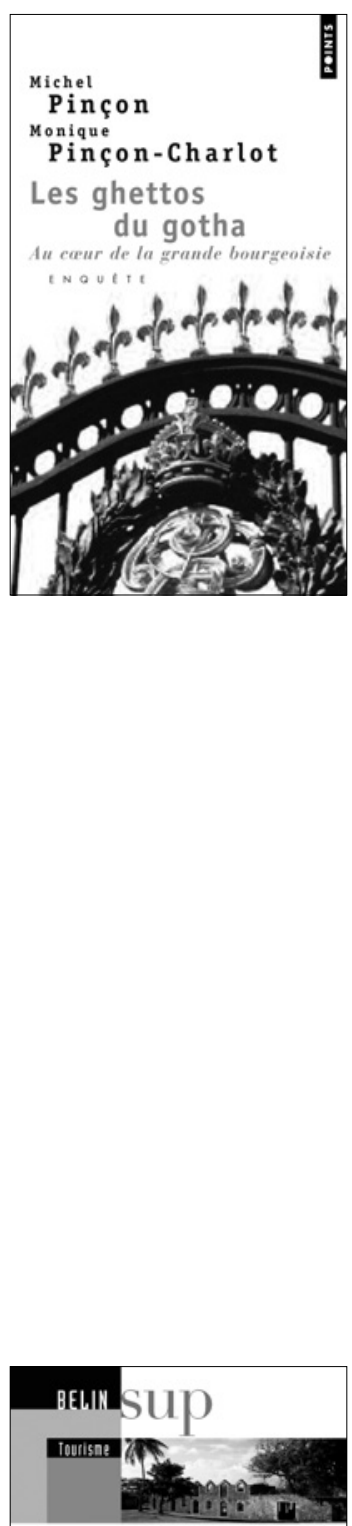

Patrimoine et tourisme Histoires, lieux, acteurs, enjeux onmensonen

\section{Les ghettos du gotha. Comment la bourgeoisie défend ses espaces}

Michel PINÇON et Monique PINÇON-CHARLOT

Paris : Seuil, 2007, 203 pages

ASIN : B005QDPONO

Les présidents de la Société pour la protection des paysages et de l'esthétique de la France (créée en 1901), de la Sauvegarde de l'art français (1921), de la Demeure historique (1924), de la Ligue urbaine et rurale (1946), des Vieilles Maisons françaises (1958), des Maisons paysannes (1965), de l'association REMPART (1966) et de la Fédération Patrimoine-Environnement (1967) se rencontrent une fois par mois pour discuter des dossiers importants. Depuis 2005, ils rencontrent le ministre de la Culture au moins deux fois par année, mais souvent davantage. Ces associations, toutes reconnues d'utilité publique, forment en quelque sorte le G8 du patrimoine en France. Elles ont toutes en commun d'être associées avec la grande bourgeoisie française.

Les sociologues Michel Pinçon et Monique Pinçon-Charlot, chercheurs au CNRS, signent un ouvrage fascinant. Ils poursuivent ainsi une œuvre entreprise à la fin des années 1980 avec le premier d'une série de plus d'une dizaine d'ouvrages sur le thème de la grande bourgeoisie. Malgré une retraite de la vie universitaire il y a près de cinq ans, ils poursuivent leur travail de recherche et de publication. Au sein de cette production, Les ghettos du gotha occupe une place particulière pour notre propos, car il traite des lieux de vie de la grande bourgeoisie et de la mobilisation déployée pour les défendre et les sauvegarder, cela souvent au nom du patrimoine. Assurément, ce sont des «militants peu ordinaires», comme le notent les auteurs, qui nous sont présentés.

L'un des intérêts de cet ouvrage est de nous entraîner dans un univers peu familier de l'investissement associatif. L'action menée par ces riches bourgeois ou ces familles de l'ancienne noblesse révèle la perméabilité de la frontière entre des intérêts privés et ceux invoqués dans la sauvegarde d'un patrimoine collectif. Comment ne pas rester songeur devant le geste de ce propriétaire qui vend les trois kilomètres de plage bordant son domaine au Conservatoire du littoral pour les soustraire à toute menace de développement? Certes, la bande riveraine est ainsi protégée, mais l'ancien propriétaire, qui ne pouvait en empêcher l'usage public, est dès lors exempté de son entretien et de celui des chemins qui y mènent. On y découvre également une volonté bien réelle de travailler à la mise en valeur du patrimoine par la recherche de financement, par la restauration, par l'organisation d'activités et par l'ouverture des lieux aux visiteurs. De lecture agréable, entre l'ouvrage grand public et une démarche universitaire, le livre de Michel Pinçon et Monique Pinçon-Charlot permet de pénétrer un monde qui n'ouvre pas facilement ses portes. Il est également riche d'enseignements pour aborder les relations entre le patrimoine et le milieu associatif.

\section{Patrimoine et tourisme : histoires, lieux, acteurs, enjeux} Olivier LAZZAROTTI

Paris : Bélin (coll. : Sup Tourisme), 2011, 302 pages

ISBN-10 : 2701149533; ISBN-13 : 978-2701149530

Un nouveau titre de la collection pour les études supérieures de Bélin vient de s'ajouter avec la publication de l'ouvrage Patrimoine et tourisme. On se réjouit de voir paraître un ouvrage scientifique de qualité qui explore les liens - j'oserais dire ontologiques - entre deux problématiques, désormais incontournables dans nos sociétés actuelles : le patrimoine et le tourisme. Olivier Lazarrotti, géographe et professeur à l'université de Picardie-Jules-Verne, livre ici le produit d'une réflexion entamée depuis plusieurs années. Cinq chapitres structurent l'ouvrage dont un est dédié aux acteurs, parmi lesquels l'auteur aborde la présence des associations pour la sauvegarde du patrimoine.

À partir de cinq études de cas, l'auteur pose certaines bases de l'action de la société civile. De l'association internationale à l'intervention extrêmement localisée, on voit apparaître des formes variées d'organisation et d'intervention qui ont comme point commun d'exister grâce au bénévolat. Certes, il s'agit d'une toute petite section dans l'ouvrage de Lazzarotti, mais elle a le mérite de souligner l'apport incontournable des associations locales, comme l'avait fait, il y a presque dix ans, l'ouvrage de Hervé Glevarec et de Guy Saez, Le patrimoine saisi par les associations (Paris : La documentation française, 2002). Il faut espérer que ce type d'études et de publications se multiplie au cours des prochaines années.

Martin DROUIN, Ph.D., Professeur, Département d'études urbaines et touristiques (ESG-UQAM) 